\title{
(1) orru A Basic Model Proposal for Memory Systems
Constructed by a MATLAB Based Neural Dynamics Simulator \\ METU
}

Önen, M. [1], Ulusoy, i. [1],[2]

[1] Department of Electrical and Electronics Engineering, METU, Ankara, Turkey

[2] Neuroscience and Neuroengineering, METU, Ankara, Turkey

\section{Introduction and Theory}

The aim of the project was to propose a generic framework for associative memory systems in intrinsic neural circuits. A MATLAB based simulator, Mnemosyne, was constructed in accordance with neurophysiology literature. Proposed model and framework were tested in memory tasks such as distinction, completion and deduction.

Principal property of the memory systems, learning, was modelled as the ability of defining a system even with a subset of its components. To conduct this feature three main functions, pattern separation, pattern integration and pattern completion, were coordinately implemented. To match these functions two main units, temporal checker disks and chaotic itinerant recall processors, were created.
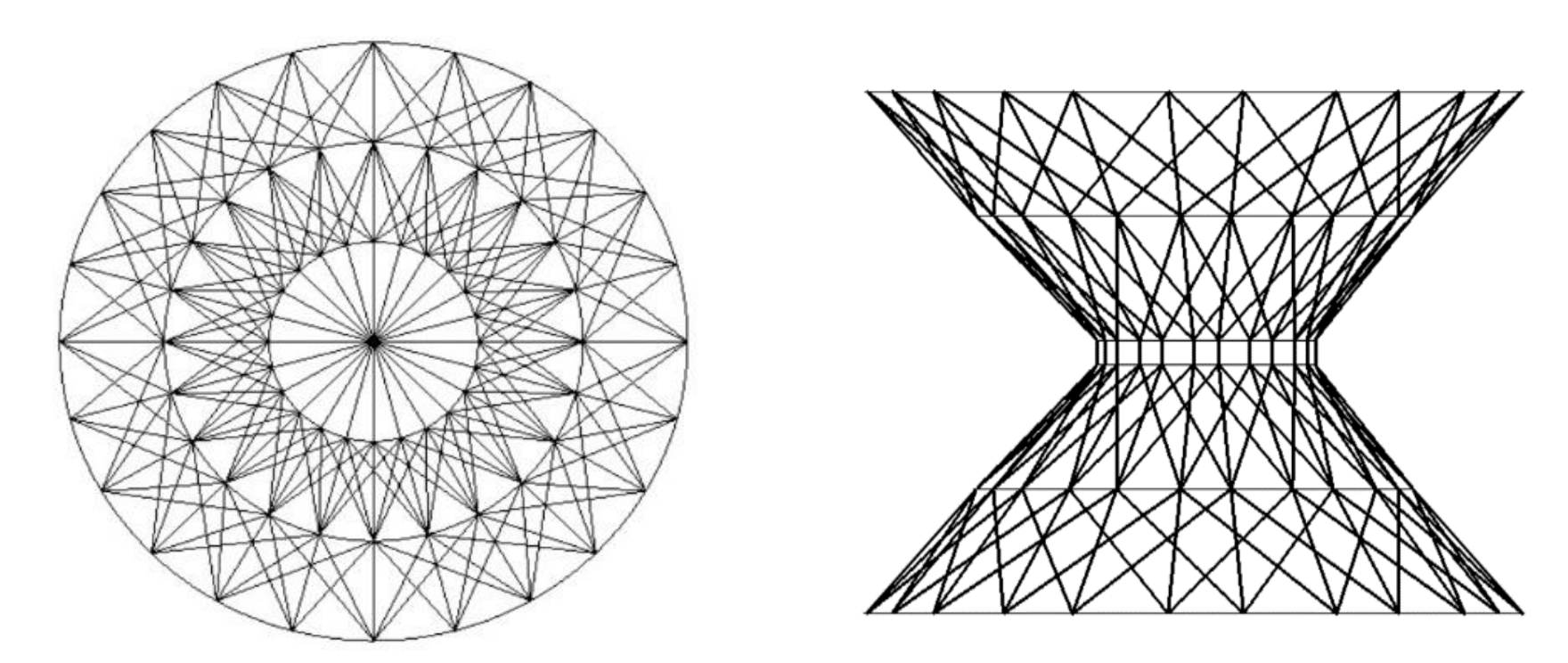

Figure 1: Illustration of temporal checker disk and their connections used in Mnemosyne

It was postulated that human brain has an evolutionary library that enables translating exteroceptive stimuli and processing more complex information internally. Translated input is fed into an initial layer and 'integrate and fire' phenomenon takes place. Neurons whose potentiation surpasses their thresholds fire through their afferent synapses. A large matrix in the simulator contains the information relating the properties of each neuron and synapse such as threshold, potentiation, time constant (capacitance), readily releasable vesicle pool (RRVP) volume and filling rate and synaptic characteristics (weight and type). Weights of the connections are updated with respect to Hebbian model, throughout the training session. Learning is conducted through a diminution in entropy. Increased determinism allows a proper firing rhythm (due to a balance in excitation and inhibition) and convergence to a set of neurons in the higher levels.

Once the system was trained with an input set, recalling through the clues (sub-contents of the trained stimuli) were conducted by a hypothetical system of chaotic itinerant bursts. If the clue is strong enough to initiate a recalling procedure, random spikes are generated at the uppermost layer of the system. At the matching areas of random bursts and incoming spikes, missing information is recreated through the distributed connection data, ending up in completing the input set. If no such initiation occurs, novelty is sensed and a new information encoding process begins.

By altering the types (static or dynamic) and values (high or low) of these properties in a designed connections diagram, features of the system (speed, capability, accuracy etc.) can be altered.

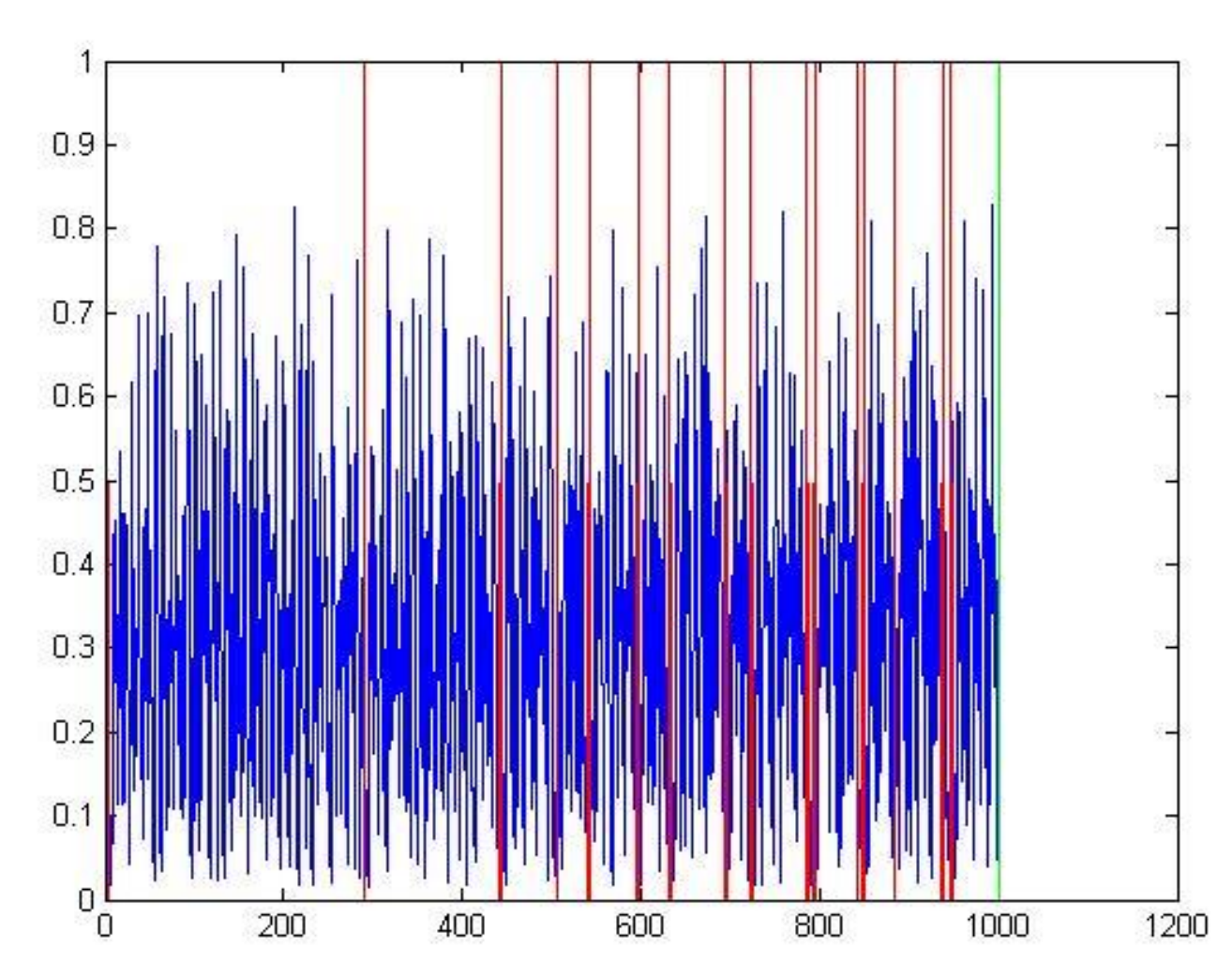

\section{Simulations and Results}

The architecture used in the simulator compels two strict rules. Since the system is frequency based, data that a spiking rhythm represents should carry an information that is not binary but consisting of many gradual levels (e.g.. contrast, sound frequency). Secondly as essence of stochastic behavior (implemented by randomizing the queuing of the signals to be processed) plays crucial role in having no bias.

Two main experiments were designed to show theory and framework's potency. In the first experiment, an image (Figure 4, top left) was trained to the system in a turn based manner. System's response to the complete and damaged inputs showed that the neural circuitry was able to correct minor flaws by using the information embedded in its connections. Experiments also showed that the algorithm was able to identify familiar object(s) in a data chunk (Figure 5).

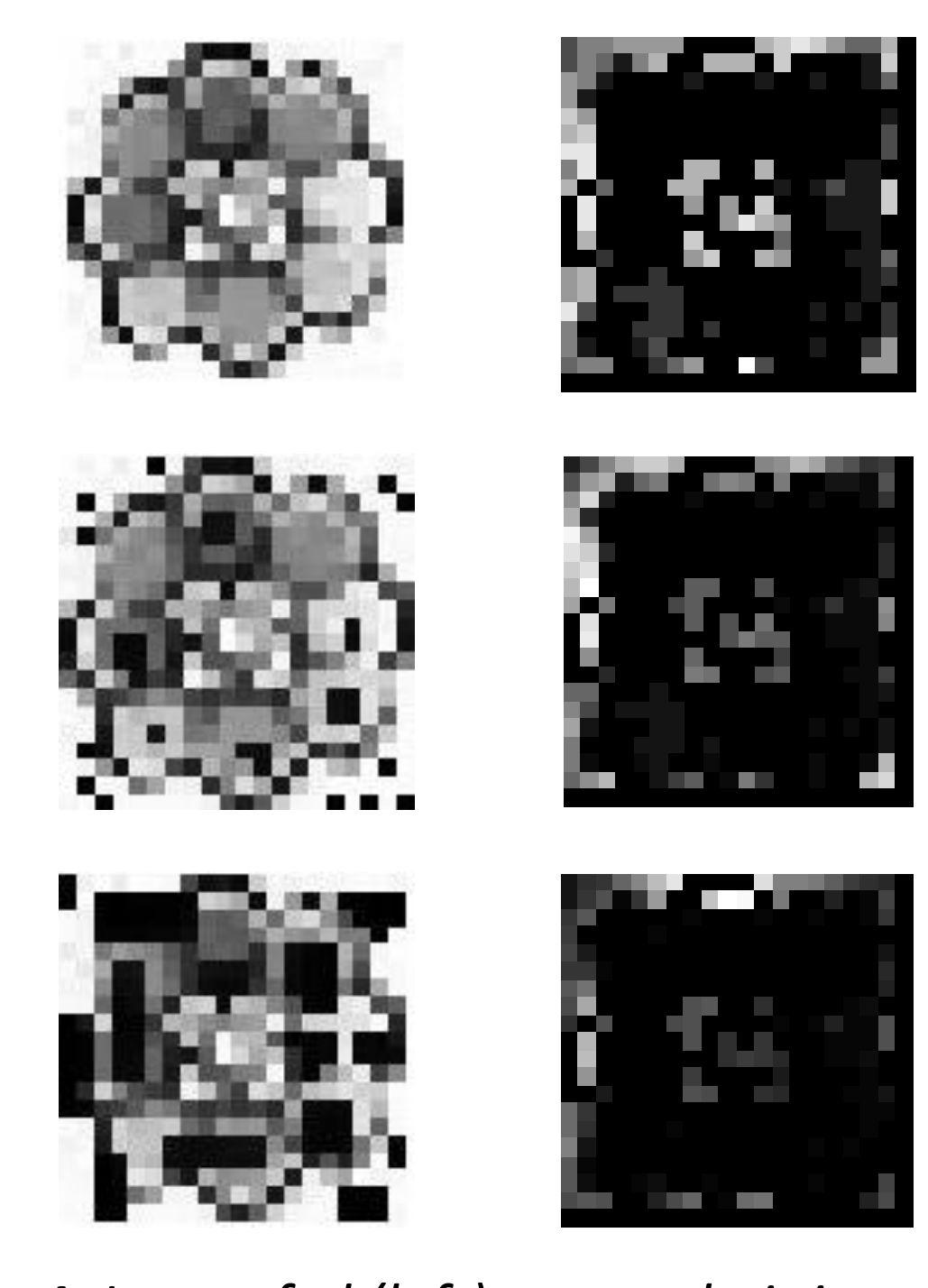

Figure 4: Inputs fed (left) versus their internal representations (right) in complete and damaged versions

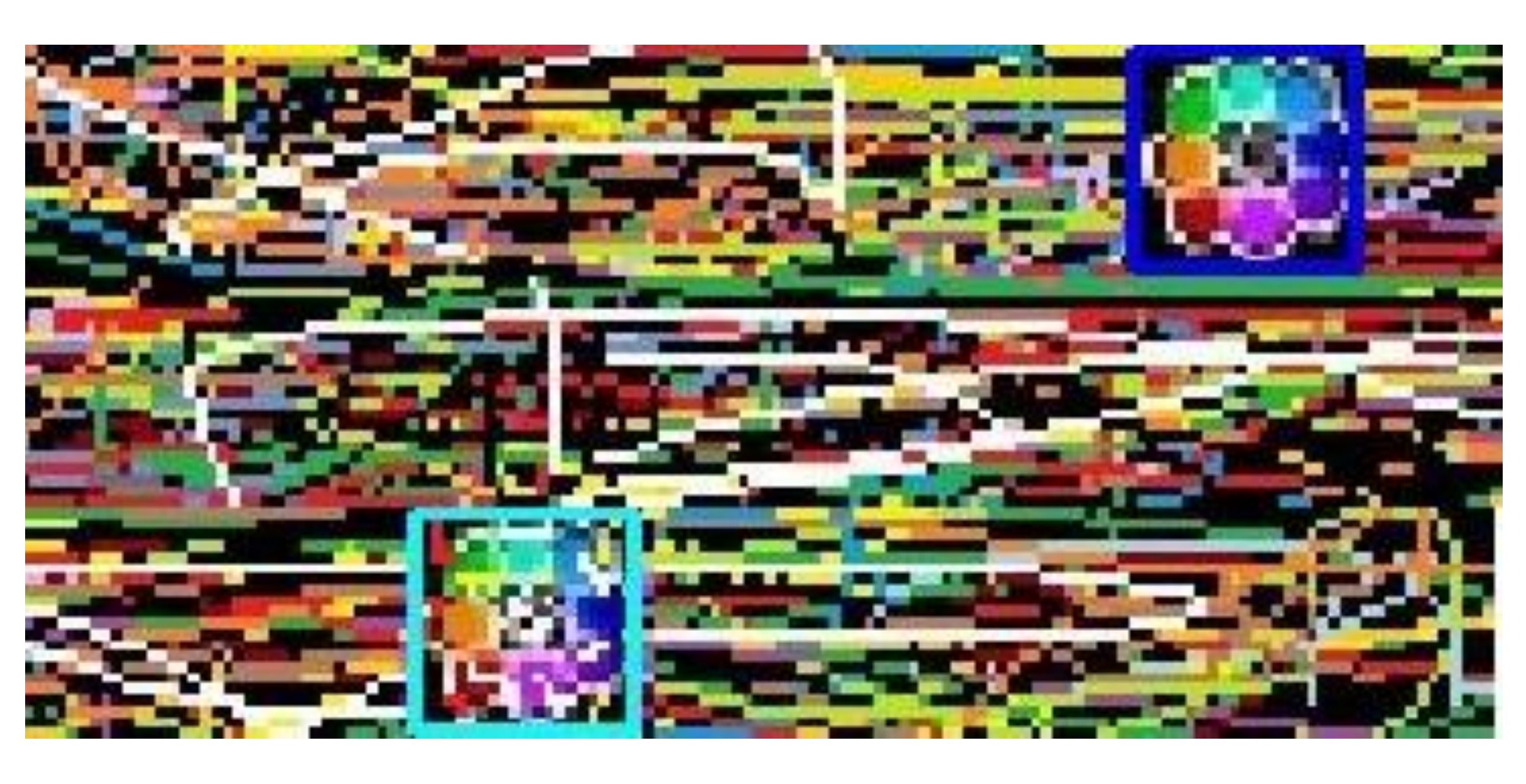

Figure 5: Correct matching of exact (dark blue) and damaged (cyan) input in a random maze
Second experiment used features (colors, shapes etc.) as its basic unit of information instead of pixels. For this experiment a regular card deck (excluding 10,J,Q and K) was trained into the system one by one in the same way as the first experiment was conducted. At the end of this procedure Mnemosyne created a database of 20 trained properties (e.g. shape of the numbers and card types) that can define all features of a card. Following the training session, another node (representing a new property) that is connected to all formerly trained properties was fired externally by the user (supervised learning) while the algorithm analyzed and detected the pre-trained features of randomly selected card duos from a subset of the deck (A to 4, all types). The machine was expected to find the set of conditions that induces the new node to be fired from a small set and apply the same rule to the complete deck (i.e. find the card duos that follow the rule but the code has never been 'told' so before). Results shown in Figure 6 confirmed Mnemosyne's ability to form complex data from basic ones and deduce accurately.

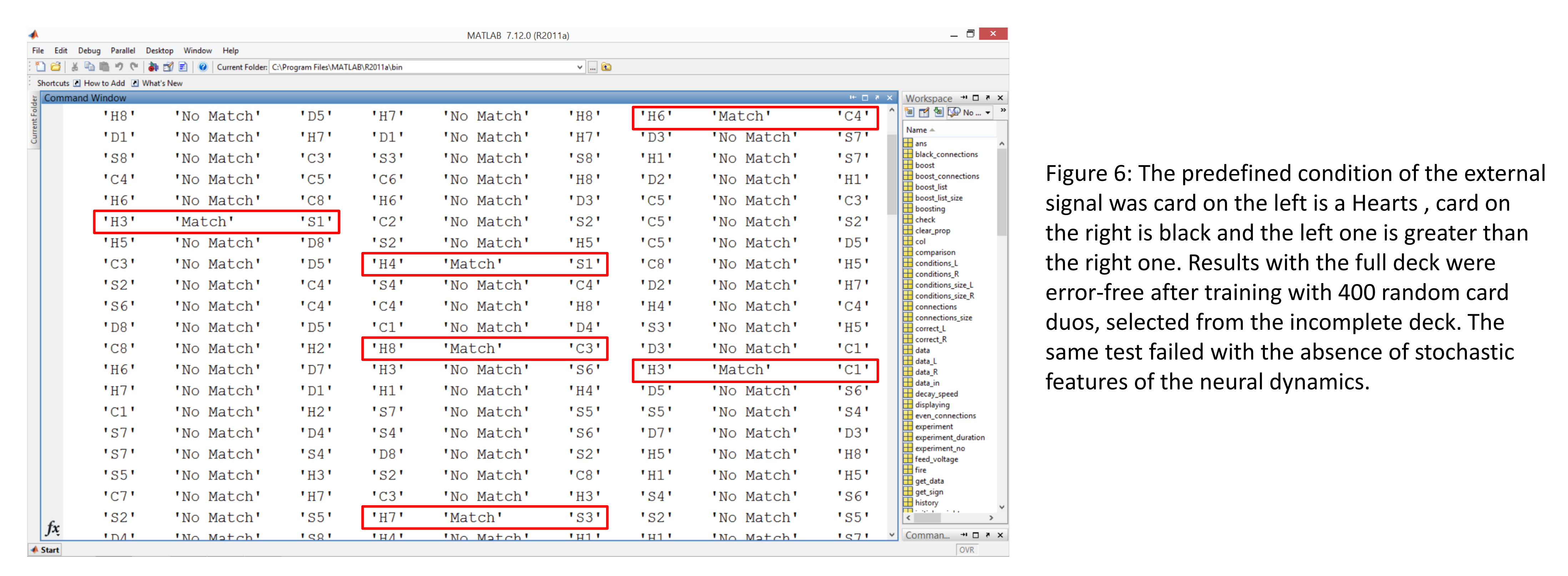

\section{Conclusions}

This project proposed a basic associative memory model and tested its capabilities. Through the sparse encoding procedure with spike trains, trained neural architecture succeeded in matching the trained pattern in a data chunk, even when it had minor flows. Furthermore it managed to define properties and gave them meanings by processing correlation information of coincidental pulses. The main philosophy of 'vast number of simple processing elements' was demonstrated to be capable of showing basic features of associative learning. Further development of the algorithm may be useful in building neuromorphic artificial intelligence systems and can be used as an experimental environment for constructing ideas in theoretical neuroscience.

\section{References}

Kesner, R. P., \& Rolls, E. T. (2015). A computatior to

Moscovitch, M. (2008). The hippocampus as a 'stupid', domain-specific module: Implications for theories of recent and remo memory, and of imagination. Canadian Journal of Experimental Psychology, 62(1), 62-79. 